\title{
Robust Filtering for Networked Stochastic Systems Subject to Sensor Nonlinearity
}

\author{
Guoqiang Wu, ${ }^{1}$ Jianwei Zhang, ${ }^{2}$ and Yuguang Bai ${ }^{1}$ \\ ${ }^{1}$ State Key Laboratory of Structural Analysis for Industrial Equipment, Dalian University of Technology, Dalian, \\ Liaoning 116024, China \\ ${ }^{2}$ School of Electronic Science and Technology, Dalian University of Technology, Dalian, Liaoning 116024, China
}

Correspondence should be addressed to Guoqiang Wu; wgq2011dllg@yahoo.com.cn

Received 4 November 2012; Accepted 19 December 2012

Academic Editor: Ligang Wu

Copyright (C) 2013 Guoqiang Wu et al. This is an open access article distributed under the Creative Commons Attribution License, which permits unrestricted use, distribution, and reproduction in any medium, provided the original work is properly cited.

\begin{abstract}
The problem of network-based robust filtering for stochastic systems with sensor nonlinearity is investigated in this paper. In the network environment, the effects of the sensor saturation, output quantization, and network-induced delay are taken into simultaneous consideration, and the output measurements received in the filter side are incomplete. The random delays are modeled as a linear function of the stochastic variable described by a Bernoulli random binary distribution. The derived criteria for performance analysis of the filtering-error system and filter design are proposed which can be solved by using convex optimization method. Numerical examples show the effectiveness of the design method.
\end{abstract}

\section{Introduction}

In recent years, networked control systems (NCSs) have been extensively investigated due to thier broad applications in industrial engineering [1]. NCSs hold a few excellent advantages such as reduction of costs of cables and power, simplification of the installation and maintenance of the whole system, and increase of the reliability [2]. However, the insertion of the communication channels also arises some unexpected phenomenon in NCSs such as signals quantization [1], intermittent data packet losses, and the signal-transmission delay $[3,4]$. These phenomena emerging in NCSs are known to be the main causes for the performance deterioration or even the instability of the controlled networked system. Over the past few years, intensive research interest has been reported in a wealth of the literature focusing on the control and filtering problems of NCSs involved with networked-induced time delay, packet losses, and signal quantization (see, e.g., [1] and the references therein).

Stochastic phenomenon frequently exhibits in many branches of science and engineering applications [5-10]. In the past few years, increasing research interests have recently been paid to the study of control and filtering problems for various continuous-time or discrete-time stochastic systems [5]. For instance, the $H_{\infty}$ nonlinear filtering problem has been investigated for discrete-time stochastic systems subject to signal quantization in [2]. The design problem of state estimation and stabilization for a nonlinear networked control systems has been addressed in [11], while the $H_{\infty}$ output feedback control problem has been considered in [12].

In practical physical systems, sensors and actuators cannot always provide unlimited amplitude signal mainly due to the physical or safety constraints [13]. The phenomenon of sensor and/or actuator saturation can yield significant limitations on various aspects of sensor and/or actuator performance, for example, the range limitations that results in the nonlinear characteristic of sensors and/or actuator $[13,14]$. For actuator saturation, a great deal of attention has been focused on the control and filtering problems for various types of systems [15]. In particular, the control problem has been investigated for continuous-time linear delay systems subject to quantization and saturation in [16], where both quantized state and quantized input are taken into consideration. 


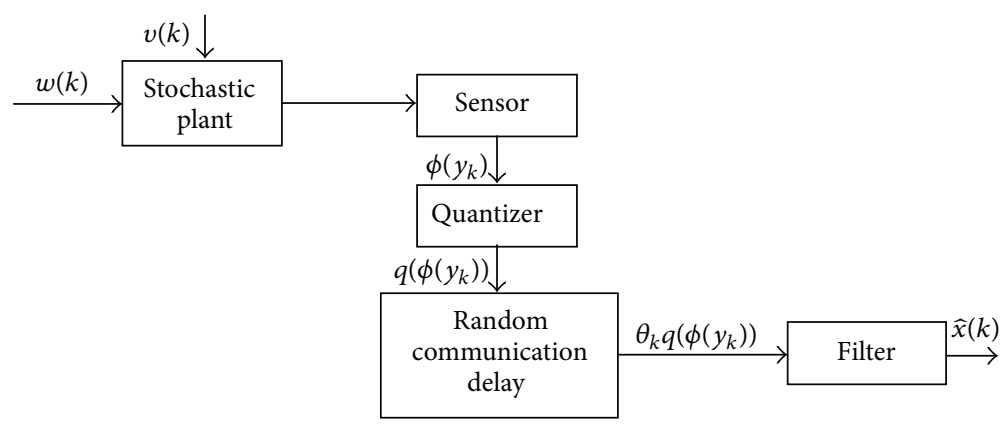

FIGURE 1: The structure of networked filtering systems.

It should be pointed out that, if we consider the filtering problem for stochastic systems in a realistic networked environment, the effects of sensor saturation, sensor quantization, and random communication delay always exhibit simultaneously. However, in networked environments, the sensor saturation may occur to be involved with state-dependent disturbance, and it may result from random sensor failures leading to intermittent saturation, sensor aging resulting in changeable saturation level, repairs of partial components, changes in the interconnections of subsystems, and so forth. Therefore, when investigating the filtering problems of NCSs with a stochastic plant, the model under consideration should be more comprehensive to reflect the realities such as the the state-dependent stochastic disturbances, the coupling effects of sensor saturation, output quantization, and networkedinduced transmission delay. Unfortunately, however, to the best of the authors' knowledge, the $H_{\infty}$ filtering problem on stochastic systems subject to sensor saturation, quantization, and random communication delay has not been investigated and remains to be important and challenging. This motivates our current work.

In this paper, we are concerned with the filter design problem for discrete-time networked stochastic systems subject to output saturation, quantization, and random communication delay. The networked-induced communication delay phenomena are modeled by a Bernoulli random binary distributed white sequence with a known conditional probability. In this network setting, the effects of sensor saturation, output quantization, and communication delay in the digital communication channel exhibit simultaneously, and the signal received in the filter side is imperfect. The objective is to analyze and design a robust filter such that the asymptotic estimates of system states are obtained by employing the incomplete output measurements. Moreover, sufficient conditions will be proposed such that the derived filtering error system is robustly stochastically stable with a prescribed disturbance attenuation level. Finally, a numerical example is provided to illustrate the effectiveness of the proposed filtering design approach.

Throughout the paper, $\mathbb{E}\{\cdot\}$ is the mathematical expectation. $\|\cdot\|$ denotes the Euclidean norm of a vector. Given a symmetric matrix $A$, the notation $A>0(<0)$ denotes a positive definite matrix (negative definite, resp.). $I_{n}$ denotes an identity matrix with dimension $n$.

\section{Problem Description}

We consider the following discrete-time stochastic system with state-dependent disturbance:

$$
\begin{gathered}
x(k+1)=A x(k)+B v(k)+[E x(k)+G v(k)] w(k), \\
y(k)=C x(k), \\
y_{\phi}(k)=\phi(y(k)), \\
y_{q}(k)=q\left(y_{\phi}(k)\right), \\
z(k)=L x(k),
\end{gathered}
$$

where $x(k) \in \mathbb{R}^{n}$ is the state; $y(k) \in \mathbb{R}^{p}$ is the output; the saturation function $\phi(\cdot)$ is defined as in $(3) ; y_{q}(k) \in \mathbb{R}^{p}$ is the quantized output, and $q(\cdot)$ is the logarithmic quantizer defined in (6)-(7); $z(k) \in \mathbb{R}^{r}$ is the state combination to be estimated; $A \in \mathbb{R}^{n \times n}, B \in \mathbb{R}^{n \times m}, C \in \mathbb{R}^{p \times n}, E \in \mathbb{R}^{n \times n}$, $G \in \mathbb{R}^{n \times m}$, and $L \in \mathbb{R}^{r \times n}$ are known constant matrices.

In plant $(1), w(k)$ is a standard one-dimensional random process on a probability space $(\Omega, \mathscr{F}, \mathscr{P})$, where $\Omega$ is the sample space, $\mathscr{F}$ is the $\sigma$-algebra of subsets of the sample space, and $\mathscr{P}$ is the probability measure on $\mathscr{F}$. The sequence of $w(k)$ is generated by $(w(k))_{k \in \mathbb{N}}$ where $\mathbb{N}$ denotes the set of natural numbers, and it satisfies that $\mathbb{E}\{w(k)\}=0, \mathbb{E}\left\{w(k)^{2}\right\}=$ $1, \mathbb{E}\{w(i) w(j)\}=0$ for $i \neq j$.

Besides, it is assumed that the exogenous disturbance $v(k) \in \mathbb{R}^{m}$ belongs to $\mathscr{L}_{E_{2}}\left([0, \infty) ; \mathbb{R}^{m}\right)$, where $\mathscr{L}_{E_{2}}\left([0, \infty) ; \mathbb{R}^{m}\right)$ denotes the space of $k$-dimensional nonanticipatory square-integrable process $\varphi(\cdot)=(\varphi(k))_{k \in \mathbb{N}}$ on $\mathbb{N}$ with respect to $\left(\varphi_{k}\right)_{k \in \mathbb{N}}$, and $\varphi(\cdot)$ satisfies

$$
\|\varphi\|_{E_{2}}^{2}=\mathbb{E}\left\{\sum_{k=0}^{\infty}\|\varphi(k)\|^{2}\right\}=\sum_{k=0}^{\infty} \mathbb{E}\left\{\|\varphi(k)\|^{2}\right\}<\infty .
$$

Remark 1. As seen in plant (1), the phenomena of sensor quantization and saturation are taken into consideration simultaneously, which is one of the main contributions of this paper. Although there have been much of the literature devoted to quantized filtering, few of which has considered the effect of sensor saturation.

The structure of the quantized filtering system is illustrated in Figure 1. 
We denote $y(k)$ as $y_{k}$ for simplicity in the following discussion. It is assumed that the saturation function $\phi(\cdot)$ : $\mathbb{R}^{p} \rightarrow \mathbb{R}^{p}$ in (1) belongs to $\left[K_{1}, K_{2}\right]$ for some given diagonal matrices $K_{1} \in \mathbb{R}^{p \times p}, K_{2} \in \mathbb{R}^{p \times p}$ with $K_{1} \geq 0, K_{2} \geq 0$ and $K_{2}>K_{1}$, and $\phi(\cdot)$ satisfies the following sector condition:

$$
\left(\phi\left(y_{k}\right)-K_{1} y_{k}\right)^{T}\left(\phi\left(y_{k}\right)-K_{2} y_{k}\right) \leq 0, \quad \forall y_{k} \in \mathbb{R}^{q} .
$$

In the light of (3), the nonlinear function $\phi\left(y_{k}\right)$ can be decomposed into a linear and a nonlinear part as follows:

$$
\phi\left(y_{k}\right)=\phi_{s}\left(y_{k}\right)+K_{1} y_{k}
$$

and the nonlinearity $\phi_{s}\left(y_{k}\right)$ satisfies $\phi_{s}\left(y_{k}\right) \in \Phi_{s}$, where the set $\Phi_{s}$ is defined as

$$
\Phi_{s} \triangleq\left\{\phi_{s}: \phi_{s}^{T}\left(y_{k}\right)\left(\phi_{s}\left(y_{k}\right)-K y_{k}\right) \leq 0\right\},
$$

and $K \triangleq K_{2}-K_{1}$.

In this paper, we employ the logarithmic quantizer for system (1) which is described as follows:

$$
q(\cdot)=\left[q_{1}(\cdot), q_{2}(\cdot), \ldots, q_{p}(\cdot)\right]^{T},
$$

and $q_{i}(\cdot)$ is defined as follows:

$$
\begin{aligned}
& q_{i}\left(\phi\left(y_{i}(k)\right)\right) \\
& =\left\{\begin{aligned}
\eta_{i}^{(j)} & \\
\text { if } \frac{1}{1+\delta_{i}} \eta_{i}^{(j)} & <\phi\left(y_{i}(k)\right) \\
& \leq \frac{1}{1-\delta_{i}} \eta_{i}^{(j)}, \\
\phi\left(y_{i}(k)\right) & >0, \\
0 \quad \text { if } \phi\left(y_{i}(k)\right) & =0, \\
-q_{i}\left(-\phi\left(y_{i}(k)\right)\right) & \\
\text { if } \phi\left(y_{i}(k)\right) & <0, \quad i=1,2, \ldots, p ; \\
& j= \pm 1, \pm 2, \ldots,
\end{aligned}\right.
\end{aligned}
$$

where $\delta_{i}=\left(1-\rho_{i}\right) /\left(1+\rho_{i}\right)$ are the quantizer parameters.

In fact, the logarithmic quantizer (7) can be characterized by the following form:

$$
q\left(\phi\left(y_{k}\right)\right)=\left(I_{p}+\Lambda(k)\right) \phi\left(y_{k}\right),
$$

where

$$
\begin{gathered}
\Lambda(k)=\operatorname{diag}\left\{\Lambda_{1}(k), \Lambda_{2}(k), \ldots, \Lambda_{p}(k)\right\}, \\
\Lambda_{j}(k) \in\left[-\eta_{j}, \eta_{j}\right], \quad j=1, \ldots, p .
\end{gathered}
$$

In this filtering problem, the measured output received in the filter side is involved with the effects of quantization and communication delay, and it is described by

$$
y_{f}(k)=\left(1-\theta_{k}\right) q\left(\phi\left(y_{k}\right)\right)+\theta_{k} q\left(\phi\left(y_{k-1}\right)\right)+D v(k) \text {, }
$$

with $D \in \mathbb{R}^{p \times m}$, and the stochastic variable $\theta_{k} \in \mathbb{R}$ is a Bernoulli distributed white sequence with the probability distribution as follows:

$$
\begin{gathered}
\operatorname{Prob}\left\{\theta_{k}=1\right\}=\mathbb{E}\left\{\theta_{k}\right\}=\bar{\theta}, \\
\operatorname{Prob}\left\{\theta_{k}=0\right\}=1-\mathbb{E}\left\{\theta_{k}\right\}=1-\bar{\theta}, \\
\operatorname{var}\left\{\theta_{k}\right\}=\mathbb{E}\left\{\left(\theta_{k}-\bar{\theta}\right)^{2}\right\}=(1-\bar{\theta}) \bar{\theta}=\theta_{1}^{2},
\end{gathered}
$$

where $0 \leq \bar{\theta}<1$ is a known positive constant to denote the probability that the packet will be transmitted successfully from sensor to filter, and $0 \leq \theta_{1}<1$ denotes the variance of $\theta_{k}$.

For simplicity, we denote $x_{k}:=x(k), f_{k}:=f\left(k, x_{k}\right), \omega_{k}:=$ $\omega(k), v_{k}:=v(k), \Lambda(k):=\Lambda_{k}$ in the following discussion. In the light of (4), (10) can be written as

$$
\begin{aligned}
y_{f}(k)= & \left(1-\theta_{k}\right)\left(I_{p}+\Delta_{k}\right)\left(\phi_{s}\left(y_{k}\right)+K_{1} C x_{k}\right) \\
& +\theta_{k}\left(I_{p}+\Delta_{k}\right)\left(\phi_{s}\left(y_{k-1}\right)+K_{1} C x_{k-1}\right)+D v_{k} .
\end{aligned}
$$

The main objective of this paper is to address the filtering problem for stochastic system (1) by employing the incomplete measurements $y_{f}(k)$. To this end, we consider the following filter of full order $n$ :

$$
\begin{gathered}
\widehat{x}(k+1)=\widehat{A} \hat{x}(k)+\widehat{B} y_{f}(k), \quad \widehat{x}(k)=0, \\
\widehat{z}(k)=L \widehat{x}(k),
\end{gathered}
$$

where $\widehat{x}(k) \in \mathbb{R}^{p}$ is the state of the filter, and $\widehat{z}(k) \in \mathbb{R}^{r}$ is the estimated signal; $\widehat{A} \in \mathbb{R}^{n \times n}$ and $\widehat{B} \in \mathbb{R}^{n \times m}$ are filter gains to be designed. 
We define the following error variables:

$$
e_{x}(k) \triangleq x(k)-\widehat{x}(k) .
$$

Subtracting (13) from (1) and considering the imperfect output measurements (12), we obtain the filtering error dynamics as follows:

$$
\begin{aligned}
e_{x}(k+1)= & {\left[A-\widehat{A}-\left(I_{p}+\Delta_{k}\right) \widehat{B} K_{1} C\right] x_{k} } \\
& +\widehat{A} e_{x}(k)-\widehat{B}\left(I_{p}+\Delta_{k}\right) \phi_{s}\left(y_{k}\right) \\
& +\left[E x_{k}+G v_{k}\right] \omega_{k}-\widehat{B} D v_{k} \\
& +\widehat{B}\left(\theta_{k}-\bar{\theta}\right)\left(I_{p}+\Delta_{k}\right)\left[\phi_{s}\left(y_{k}\right)+K_{1} C x_{k}\right] \\
& +\widehat{B} \bar{\theta}\left(I_{p}+\Delta_{k}\right)\left[\phi_{s}\left(y_{k}\right)+K_{1} C x_{k}\right] \\
& -\widehat{B}\left(\theta_{k}-\bar{\theta}\right)\left(I_{p}+\Delta_{k-1}\right)\left[\phi_{s}\left(y_{k-1}\right)+K_{1} C x_{k-1}\right] \\
& -\widehat{B} \bar{\theta}\left(I_{p}+\Delta_{k-1}\right)\left[\phi_{s}\left(y_{k-1}\right)+K_{1} C x_{k-1}\right] .
\end{aligned}
$$

We define the following error variables:

$$
e(k) \triangleq\left[x_{k}^{T}, e_{x}^{T}(k)\right]^{T}, \quad e_{z}(k) \triangleq z(k)-\widehat{z}(k) .
$$

Combining the error dynamics (15) with the plant (1), we obtain the following augmented filtering error dynamics:

$$
\begin{aligned}
e(k+1)= & \left(\bar{A}_{1}+\Delta \bar{A}_{1}\right) e_{k}+\left(\bar{H}_{1}+\Delta \bar{H}_{1}\right) \phi_{s}\left(y_{k}\right)+\bar{B}_{1} v_{k} \\
& +\left(\theta_{k}-\bar{\theta}\right) \\
& \times\left\{\left(\bar{A}_{2}+\Delta \bar{A}_{2}\right) e_{k}+\left(\bar{H}_{2}+\Delta \bar{H}_{2}\right) \phi_{s}\left(y_{k}\right)\right\} \\
& +\left(\theta_{k}-\bar{\theta}\right) \\
& \times\left\{\left(\bar{A}_{3}+\Delta \bar{A}_{3}\right) e_{k-1}+\left(\bar{H}_{3}+\Delta \bar{H}_{3}\right) \phi_{s}\left(y_{k-1}\right)\right\} \\
& +\left(\bar{H}_{4}+\Delta \bar{H}_{4}\right) \phi\left(y_{k-1}\right)+\left(\bar{A}_{4}+\Delta \bar{A}_{4}\right) e_{k-1} \\
& +\left(\bar{E} e_{k}+\bar{G} v_{k}\right) \omega_{k}, \\
& \quad e_{z}(k)=\bar{L} e(k),
\end{aligned}
$$

where

$$
\begin{aligned}
& \bar{A}_{1}=\left[\begin{array}{cc}
A, & 0 \\
A-\widehat{A}-\bar{\theta} \widehat{B} K_{1} C, & \widehat{A}
\end{array}\right], \quad \bar{A}_{2}=\left[\begin{array}{cc}
0, & 0 \\
\widehat{B} K_{1} C, & 0
\end{array}\right], \\
& \bar{A}_{3}=\left[\begin{array}{cc}
0, & 0 \\
-\widehat{B} K_{1} C, & 0
\end{array}\right], \quad \bar{A}_{4}=\left[\begin{array}{cc}
0, & 0 \\
-\bar{\theta} \widehat{B} K_{1} C, & 0
\end{array}\right], \\
& \bar{B}_{1}=\left[\begin{array}{c}
B \\
\widehat{B} D
\end{array}\right], \quad \bar{B}_{3}=\left[\begin{array}{c}
0 \\
-\widehat{B} D
\end{array}\right], \quad \bar{B}_{4}=\left[\begin{array}{c}
0 \\
-\bar{\theta} \widehat{B} D
\end{array}\right], \\
& \bar{H}_{1}=\left[\begin{array}{c}
0 \\
(\bar{\theta}-1) \widehat{B}
\end{array}\right], \quad \bar{H}_{2}=\left[\begin{array}{l}
0 \\
\widehat{B}
\end{array}\right], \\
& \bar{H}_{3}=\left[\begin{array}{c}
0 \\
-\widehat{B}
\end{array}\right], \quad \bar{H}_{4}=\left[\begin{array}{c}
0 \\
-\bar{\theta} \widehat{B}
\end{array}\right] \text {, } \\
& \Delta \bar{A}_{1}=\left[\begin{array}{cc}
A, & 0 \\
(\bar{\theta}-1) \widehat{B} \Lambda_{k} K_{1} C, & 0
\end{array}\right], \quad \Delta \bar{A}_{2}=\left[\begin{array}{cc}
0, & 0 \\
\widehat{B} \Lambda_{k} K_{1} C, & 0
\end{array}\right], \\
& \Delta \bar{A}_{3}=\left[\begin{array}{cc}
0, & 0 \\
-\widehat{B} \Lambda_{k} K_{1} C, & 0
\end{array}\right], \quad \Delta \bar{A}_{4}=\left[\begin{array}{cc}
0, & 0 \\
-\bar{\theta} \widehat{B} \Lambda_{k} K_{1} C, & 0
\end{array}\right], \\
& \Delta \bar{B}_{3}=\left[\begin{array}{c}
0 \\
-\widehat{B} \Lambda_{k-1} D
\end{array}\right], \quad \Delta \bar{B}_{4}=\left[\begin{array}{c}
0 \\
-\bar{\theta} \widehat{B} \Lambda_{k-1} D
\end{array}\right], \\
& \Delta \bar{H}_{1}=\left[\begin{array}{c}
0 \\
(\bar{\theta}-1) \widehat{B} \Lambda_{k}
\end{array}\right], \quad \Delta \bar{H}_{2}=\left[\begin{array}{c}
0 \\
\widehat{B} \Lambda_{k}
\end{array}\right] \text {, } \\
& \Delta \bar{H}_{3}=\left[\begin{array}{c}
0 \\
-\widehat{B} \Lambda_{k-1}
\end{array}\right], \quad \Delta \bar{H}_{4}=\left[\begin{array}{c}
0 \\
-\bar{\theta} \widehat{B} \Lambda_{k-1}
\end{array}\right], \\
& \bar{E}=\left[\begin{array}{cc}
E & 0 \\
E & 0
\end{array}\right], \quad \bar{G}=\left[\begin{array}{l}
G \\
G
\end{array}\right], \\
& \bar{L}=[0, L] \text {. }
\end{aligned}
$$

For simplicity, we denote

$$
\begin{array}{cc}
\widetilde{A}_{1}:=\bar{A}_{1}+\Delta \bar{A}_{1}(k), & \widetilde{A}_{2}:=\bar{A}_{2}+\Delta \bar{A}_{2}(k), \\
\widetilde{A}_{3}:=\bar{A}_{3}+\Delta \bar{A}_{3}(k), & \widetilde{A}_{4}:=\bar{A}_{4}+\Delta \bar{A}_{4}(k), \\
\widetilde{B}_{3}:=\bar{B}_{3}+\Delta \bar{B}_{3}(k), & \widetilde{B}_{4}:=\bar{B}_{4}+\Delta \bar{B}_{4}(k), \\
\widetilde{H}_{1}:=\bar{H}_{1}+\Delta \bar{H}_{1}(k), & \widetilde{H}_{2}:=\bar{H}_{2}+\Delta \bar{H}_{2}(k), \\
\widetilde{H}_{3}:=\bar{H}_{3}+\Delta \bar{H}_{3}(k), & \widetilde{H}_{4}:=\bar{H}_{4}+\Delta \bar{H}_{4}(k),
\end{array}
$$

in the following discussion.

System (17) contains sector-bounded uncertainty $\Lambda_{k}$ and stochastic parameters $\theta_{k}$, and thus it is an uncertain stochastic parameter system where the uncertainties resulted from the quantization error. Due to this fact, it is required to introduce the notion of stochastic stability before proceeding with the subsequent analysis.

Before formulating the problem to be investigated, we introduce the following definition and lemma. 
Definition 2 (see [17]). The stochastic system (17) under $v(k)=0$ is said to be stochastically stable if there exists a scalar $\beta>0$ such that

$$
\mathbb{E}\left\{\sum_{k=0}^{\infty}\|x(k)\|^{2}\right\} \leq \beta \mathbb{E}\left\{\|x(0)\|^{2}\right\} .
$$

Lemma 3 (see [18]). For any real vectors $a, b$ and matrix $R>0$ of compatible dimensions, the following inequality holds:

$$
a^{T} b+b^{T} a \leq a^{T} R a+b^{T} R^{-1} b .
$$

In the sequel, the main objective of this paper is as follows.

$H_{\infty}$ Filtering Problem. Given a disturbance attenuation level $\gamma>0$, the parameters $\widehat{A}$ and $\widehat{B}$ of filter (13) are designed such that (i) the resulting filtering error system is stochastically stable for $v(k)=0$, and (ii) for any function $\phi(\cdot) \in\left[K_{1}, K_{2}\right]$, $\|z-\widehat{z}\|_{E_{2}}<\gamma\left\|v_{k}\right\|_{E_{2}}$ holds under zero initial conditions for all $\widetilde{v}(k) \in \mathscr{L}_{E_{2}}\left([0, \infty) ; \mathbb{R}^{m}\right)$.

\section{Filtering Performance Analysis}

In this section, we shall focus on the $H_{\infty}$ performance, that is, presenting sufficient conditions under which the $H_{\infty}$ performance index is achieved for a given filter.

Theorem 4. If there exist positive and definite matrices $\bar{P} \in$ $\mathbb{R}^{n \times n}$, such that the following matrix inequality constraint holds:

$\Gamma \triangleq\left[\begin{array}{ccccccc}-\bar{P}+\bar{L}^{T} \bar{L} & 0 & \bar{C}^{T} K^{T} & 0 & 0 & \widetilde{A}_{1}^{T} & \widetilde{A}_{2}^{T} \\ * & -\gamma^{2} I_{m} & 0 & 0 & 0 & \bar{B}_{1}^{T} & 0 \\ * & * & -2 I_{p} & 0 & 0 & -\widetilde{H}_{1}^{T} & \widetilde{H}_{2}^{T} \\ * & * & * & -\bar{Q} & \bar{C}^{T} K^{T} & \widetilde{A}_{4}^{T} & \widetilde{A}_{3}^{T} \\ * & * & * & * & -2 I_{p} & \widetilde{H}_{4}^{T} & \widetilde{H}_{3}^{T} \\ * & * & * & * & * & -\bar{P}^{-1} & 0 \\ * & * & * & * & * & * & -\theta_{1}^{2} \bar{P}^{-1}\end{array}\right]$
$<0$,

then the filtering error system (17) is stochastically stable.

Proof. Consider the filtering error system (17) with $v(k)=0$, select the stochastic Lyapunov functional candidate as

$$
V(e(k), k)=e^{T}(k) \bar{P} e(k)+e_{k-1}^{T} \bar{Q} e_{k-1},
$$

with $\bar{P}=\left[\begin{array}{cc}P_{1} & 0 \\ 0 & P_{2}\end{array}\right]>0, \bar{Q}=\left[\begin{array}{cc}\mathrm{Q}_{1} & 0 \\ 0 & \mathrm{Q}_{2}\end{array}\right]>0$. It follows from (17) that

$$
\begin{aligned}
\Delta V(k)=\mathbb{E} & \left\{V(e(k+1), k+1) \mid x_{k}, e_{k}\right\}-V(e(k), k) \\
= & {\left[\widetilde{A}_{1} e_{k}+\widetilde{H}_{1} \phi_{s}\left(y_{k}\right)+\widetilde{A}_{4} e_{k-1}+\widetilde{H}_{4} \phi\left(y_{k-1}\right)\right]^{T} } \\
& \times \bar{P}\left[\widetilde{A}_{1} e_{k}+\widetilde{H}_{1} \phi_{s}\left(y_{k}\right)+\widetilde{A}_{4} e_{k-1}+\widetilde{H}_{4} \phi\left(y_{k-1}\right)\right] \\
& +\left(\bar{E} e_{k}\right)^{T} \bar{P} \bar{E} e_{k}-e_{k}^{T} \bar{Q}_{k}-e_{k-1}^{T} \bar{Q} e_{k-1} \\
& +\mathbb{E}\left\{\left(\theta_{k}-\bar{\theta}\right)^{2}\right\} \\
& \times\left[\widetilde{A}_{2} e_{k}+\widetilde{H}_{2} \phi_{s}\left(y_{k}\right)+\widetilde{A}_{3} e_{k-1}+\widetilde{H}_{3} \phi\left(y_{k-1}\right)\right]^{T} \\
& \times \bar{P}\left[\widetilde{A}_{1} e_{k}+\widetilde{H}_{1} \phi_{s}\left(y_{k}\right)+\widetilde{A}_{4} e_{k-1}+\widetilde{H}_{4} \phi\left(y_{k-1}\right)\right] .
\end{aligned}
$$

Notice that $\mathbb{E}\left\{\left(\theta_{k}-\bar{\theta}\right)^{2}\right\}=\theta_{1}^{2}$, and thus we have

$$
\begin{aligned}
\Delta V(k)= & {\left[\widetilde{A}_{1} e_{k}+\widetilde{H}_{1} \phi_{s}\left(y_{k}\right)+\widetilde{A}_{4} e_{k-1}+\widetilde{H}_{4} \phi\left(y_{k-1}\right)\right]^{T} } \\
& \times \bar{P}\left[\widetilde{A}_{1} e_{k}+\widetilde{H}_{1} \phi_{s}\left(y_{k}\right)+\widetilde{A}_{4} e_{k-1}+\widetilde{H}_{4} \phi\left(y_{k-1}\right)\right] \\
& +\left(\bar{E} e_{k}\right)^{T} \bar{P}\left(\bar{E} e_{k}\right)-e_{k}^{T} \bar{Q} e_{k}-e_{k-1}^{T} \bar{Q} e_{k-1} \\
& +\theta_{1}^{2}\left[\widetilde{A}_{2} e_{k}+\widetilde{H}_{2} \phi_{s}\left(y_{k}\right)+\widetilde{A}_{3} e_{k-1}+\widetilde{H}_{3} \phi\left(y_{k-1}\right)\right]^{T} \\
& \times \bar{P}\left[\widetilde{A}_{1} e_{k}+\widetilde{H}_{1} \phi_{s}\left(y_{k}\right)+\widetilde{A}_{4} e_{k-1}+\widetilde{H}_{4} \phi\left(y_{k-1}\right)\right] .
\end{aligned}
$$

In fact, for the saturation function $\phi_{s}\left(y_{k}\right)$, from (5) we have that

$$
\begin{gathered}
-2 \phi_{s}^{T}\left(y_{k}\right) \phi_{s}\left(y_{k}\right)+2 \phi_{s}^{T}\left(y_{k}\right) K y_{k}>0, \\
-2 \phi_{s}^{T}\left(y_{k-1}\right) \phi_{s}\left(y_{k-1}\right)+2 \phi_{s}^{T}\left(y_{k-1}\right) K y_{k-1}>0,
\end{gathered}
$$

which implies that

$$
\begin{gathered}
-2 \phi_{s}^{T}\left(y_{k}\right) \phi_{s}\left(y_{k}\right)+2 \phi_{s}^{T}\left(y_{k}\right) K \bar{C} e_{k}>0, \\
-2 \phi_{s}^{T}\left(y_{k-1}\right) \phi_{s}\left(y_{k-1}\right)+2 \phi_{s}^{T}\left(y_{k-1}\right) K \bar{C} e_{k-1}>0,
\end{gathered}
$$


In the light of (26), (27), and (24), one can obtain

$$
\begin{aligned}
\Delta V(k)= & {\left[\widetilde{A}_{1} e_{k}+\widetilde{H}_{1} \phi_{s}\left(y_{k}\right)+\widetilde{A}_{4} e_{k-1}+\widetilde{H}_{4} \phi\left(y_{k-1}\right)\right]^{T} } \\
& \times \bar{P}\left[\widetilde{A}_{1} e_{k}+\widetilde{H}_{1} \phi_{s}\left(y_{k}\right)+\widetilde{A}_{4} e_{k-1}+\widetilde{H}_{4} \phi\left(y_{k-1}\right)\right] \\
& +\left(\bar{E} e_{k}\right)^{T} \bar{P}\left(\bar{E} e_{k}\right)-e_{k}^{T} \bar{Q} e_{k}-e_{k-1}^{T} \bar{Q} e_{k-1} \\
& +\theta_{1}^{2}\left[\widetilde{A}_{2} e_{k}+\widetilde{H}_{2} \phi_{s}\left(y_{k}\right)+\widetilde{A}_{3} e_{k-1}+\widetilde{H}_{3} \phi\left(y_{k-1}\right)\right]^{T} \\
& \times \bar{P}\left[\widetilde{A}_{1} e_{k}+\widetilde{H}_{1} \phi_{s}\left(y_{k}\right)+\widetilde{A}_{4} e_{k-1}+\widetilde{H}_{4} \phi\left(y_{k-1}\right)\right] \\
& -2 \phi_{s}^{T}\left(y_{k}\right) \phi_{s}\left(y_{k}\right)+e^{T}(k) \bar{C}^{T} K^{T} \phi_{s}\left(y_{k}\right) \\
& +\phi_{s}^{T}\left(y_{k}\right) K \bar{C} e(k)-2 \phi_{s}^{T}\left(y_{k-1}\right) \phi_{s}\left(y_{k-1}\right) \\
& +e_{k-1}^{T} \bar{C}^{T} K^{T} \phi_{s}\left(y_{k-1}\right)+\phi_{s}^{T}\left(y_{k-1}\right) K \bar{C} e_{k-1} .
\end{aligned}
$$

The following proof is divided into the following two parts: (i) a proof that the filtering error system (17) is stochastically stable with $v(k)=0$, and (ii) a proof that $\left\|e_{z}(k)\right\|_{E_{2}}<\gamma\left\|v_{k}\right\|_{E_{2}}$.

(i) Firstly, we establish the stochastic stability of the filtering error system (17) under the condition (22). It follows from (28) with $v_{k}=0$ that

$$
\begin{aligned}
\Delta V(k)= & {\left[\widetilde{A}_{1} e_{k}+\widetilde{H}_{1} \phi_{s}\left(y_{k}\right)+\widetilde{A}_{4} e_{k-1}+\widetilde{H}_{4} \phi\left(y_{k-1}\right)\right]^{T} } \\
& \times \bar{P}\left[\widetilde{A}_{1} e_{k}+\widetilde{H}_{1} \phi_{s}\left(y_{k}\right)+\widetilde{A}_{4} e_{k-1}+\widetilde{H}_{4} \phi\left(y_{k-1}\right)\right] \\
& +e_{k}^{T} \bar{E}^{T} \overline{P E} e_{k}-e_{k}^{T} \bar{Q} e_{k}-e_{k-1}^{T} \bar{Q} e_{k-1} \\
& +\theta_{1}^{2}\left[\widetilde{A}_{2} e_{k}+\widetilde{H}_{2} \phi_{s}\left(y_{k}\right)+\widetilde{A}_{3} e_{k-1}+\widetilde{H}_{3} \phi\left(y_{k-1}\right)\right]^{T} \\
& \times \bar{P}\left[\widetilde{A}_{1} e_{k}+\widetilde{H}_{1} \phi_{s}\left(y_{k}\right)+\widetilde{A}_{4} e_{k-1}+\widetilde{H}_{4} \phi\left(y_{k-1}\right)\right] \\
& -2 \phi_{s}^{T}\left(y_{k}\right) \phi_{s}\left(y_{k}\right)+e^{T}(k) \bar{C}^{T} K^{T} \phi_{s}\left(y_{k}\right) \\
& +\phi_{s}^{T}\left(y_{k}\right) K \bar{C} e(k)-2 \phi_{s}^{T}\left(y_{k-1}\right) \phi_{s}\left(y_{k-1}\right) \\
& +e_{k-1}^{T} \bar{C}^{T} K^{T} \phi_{s}\left(y_{k-1}\right)+\phi_{s}^{T}\left(y_{k-1}\right) K \bar{C} e_{k-1} \\
\leq & \xi^{T}(k) \Pi \xi(k),
\end{aligned}
$$

where

$$
\begin{gathered}
\xi(k) \triangleq\left[e^{T}(k), \phi_{s}^{T}\left(y_{k}\right), e^{T}(k-1), \phi_{s}^{T}\left(y_{k-1}\right)\right]^{T}, \\
\Pi \triangleq\left[\begin{array}{cccc}
\widetilde{A}_{1} & \widetilde{H}_{1} & \widetilde{A}_{4} & \widetilde{H}_{4} \\
\widetilde{A}_{2} & \widetilde{H}_{2} & \widetilde{A}_{3} & \widetilde{H}_{3}
\end{array}\right]^{T}\left[\begin{array}{cc}
\bar{P} & 0 \\
0 & \theta_{1}^{2} \bar{P}
\end{array}\right]\left[\begin{array}{cccc}
\widetilde{A}_{1} & \widetilde{H}_{1} & \widetilde{A}_{4} & \widetilde{H}_{4} \\
\widetilde{A}_{2} & \widetilde{H}_{2} & \widetilde{A}_{3} & \widetilde{H}_{3}
\end{array}\right] \\
+\left[\begin{array}{cccc}
-\bar{P}+\bar{L}^{T} \bar{L} & \bar{C}^{T} \bar{K}^{T} & 0 & 0 \\
* & -2 I_{p} & 0 & 0 \\
* & * & -Q & \bar{C}^{T} K^{T} \\
* & * & * & -2 I_{p}
\end{array}\right] .
\end{gathered}
$$

It is obvious that if $\Pi<0$, one can obtain that $\Delta V(k)<$ 0 . Therefore, it follows from Kolmanovskii and Myshkis [19] that the filtering error system (17) with $v(k)=0$ is stochastically stable. On the other hand, by means of Schur's complement, $\Pi<0$ is equivalent to the following matrix condition:

$$
\left[\begin{array}{cccccc}
-\bar{P}+\bar{L}^{T} \bar{L} & \bar{C}^{T} \bar{K}^{T} & 0 & 0 & \widetilde{A}_{1}^{T} & \widetilde{A}_{2}^{T} \\
* & -2 I_{p} & 0 & 0 & \widetilde{H}_{1}^{T} & \widetilde{H}_{2}^{T} \\
* & * & -Q & \bar{C}^{T} K^{T} & \widetilde{A}_{4}^{T} & \widetilde{A}_{3}^{T} \\
* & * & * & -2 I_{p} & \widetilde{H}_{4}^{T} & \widetilde{H}_{3}^{T} \\
* & * & * & * & -\bar{P}^{-1} & 0 \\
* & * & * & * & * & -\bar{\theta}^{2} \bar{P}^{-1}
\end{array}\right]<0 .
$$

Notice that the condition (22) can imply (31). This means that if there exist positive and definite matrices $\bar{P}$, such that matrix condition (22) holds, then system (17) is stochastically stable.

(ii) Next, the objective should be devoted to prove that the filtering error system (17) satisfies

$$
\left\|e_{z}(k)\right\|_{E_{2}}<\gamma\left\|v_{k}\right\|_{E_{2}},
$$

for all nonzero $v(k) \in \mathscr{L}_{E_{2}}\left([0, \infty) ; \mathbb{R}^{m}\right)$.

In fact, under zero initial conditions, it is shown that

$$
\mathbb{E}\{V(e(k), t)\}=\mathbb{E}\left\{\sum_{k=1}^{N} \Delta V(k)\right\} .
$$

We define the following performance index function:

$$
J(k)=\mathbb{E}\left\{\sum_{k=1}^{N}\left[e_{z}^{T}(k) e_{z}(k)-\gamma^{2} v_{k}^{T} v_{k}\right]\right\},
$$

for any integer $N$.

It follows from (34) that

$$
\begin{aligned}
J(k)= & \mathbb{E}\left\{\sum_{k=1}^{N}\left[e_{z}^{T}(k) e_{z}(k)-\gamma^{2} v_{k}^{T} v_{k}+\Delta V(k)\right]\right\} \\
& -\mathbb{E}\{V(e(k), N)\} \\
& \leq \mathbb{E}\left\{\sum_{k=1}^{N} \zeta^{T}(k) \Xi \zeta(k)\right\},
\end{aligned}
$$


where

$$
\begin{aligned}
\zeta(k) \triangleq & {\left[e^{T}(k), v^{T}(k), \phi_{s}^{T}\left(y_{k}\right), e^{T}(k-1), \phi_{s}^{T}\left(y_{k-1}\right)\right]^{T}, } \\
\Xi \triangleq & {\left[\begin{array}{ccccc}
\widetilde{A}_{1} & \widetilde{B}_{1} & \widetilde{H}_{1} & \widetilde{A}_{4} & \widetilde{H}_{4} \\
\widetilde{A}_{2} & 0 & \widetilde{H}_{2} & \widetilde{A}_{3} & \widetilde{H}_{3}
\end{array}\right]^{T}\left[\begin{array}{cc}
\bar{P} & 0 \\
0 & \theta_{1}^{2} \bar{P}
\end{array}\right] } \\
& \times\left[\begin{array}{cccccc}
\widetilde{A}_{1} & \widetilde{B}_{1} & \widetilde{H}_{1} & \widetilde{A}_{4} & \widetilde{H}_{4} \\
\widetilde{A}_{2} & 0 & \widetilde{H}_{2} & \widetilde{A}_{3} & \widetilde{H}_{3}
\end{array}\right] \\
& +\left[\begin{array}{ccccc}
-\bar{P}+\bar{L}^{T} \bar{L}^{2} & 0 & \bar{C}^{T} \bar{K}^{T} & 0 & 0 \\
* & -\gamma^{2} I_{m} & 0 & 0 & 0 \\
* & * & -2 I_{p} & 0 & 0 \\
* & * & * & -Q & \bar{C}^{T} K^{T} \\
* & * & * & * & -2 I_{p}
\end{array}\right] .
\end{aligned}
$$

From (35), it can be shown that if $\Xi<0$ holds, then $J(k)<0$, which implies that (32) holds for any nonzero $v(k) \in \mathscr{L}_{E_{2}}\left([0, \infty) ; \mathbb{R}^{m}\right)$. Furthermore, it is easy to see that $\Xi<0$ is equivalent to the condition (22). This means that the condition (22) in Theorem 4 can imply $\left\|e_{z}(k)\right\|_{E_{2}}<\gamma\|v(k)\|_{E_{2}}$. This completes the proof.

\section{4. $H_{\infty}$ Filter Design}

In this section, the attention should be paid on coping with the addressed filter design problem for the discrete-time stochastic system (1) based on Theorem 4 .

Lemma 5 (see [18]). Let $Q, R$, and $F(t)$ be real matrices of appropriate dimensions with $F(t)$ satisfying $F^{T}(t) F(t)<I$. Then, for any scalar $\varepsilon>0$,

$$
Q F(t) R+R^{T} F^{T}(t) Q^{T} \leq \varepsilon Q Q^{T}+\varepsilon^{-1} R^{T} R .
$$

The following theorem provides the sufficient LMI condition for the existence of the proposed robust filter (13).

Theorem 6. Consider the discrete-time stochastic system (1), for a given disturbance level $\gamma>0$, if there exist positive and definite matrices $X, Y \in \mathbb{R}^{n \times n}$, and matrices $W \in \mathbb{R}^{n \times n}$ and $U \in \mathbb{R}^{n \times m}$, and positive scalars $\varepsilon_{1}, \varepsilon_{2}$, and $\varepsilon_{3}$ such that the following LMI holds:

$$
\left[\begin{array}{ccc}
\Phi_{11} & \Phi_{12} & \Phi_{13} \\
* & \Phi_{22} & \Phi_{23} \\
* & * & \Phi_{33}
\end{array}\right]<0
$$

with

$$
\begin{aligned}
& \Phi_{11}=\left[\begin{array}{ccccccc}
\widetilde{\Gamma}_{11} & 0 & 0 & C^{T} K^{T} & 0 & 0 & 0 \\
* & \widetilde{\Gamma}_{22} & 0 & 0 & 0 & 0 & 0 \\
* & * & \widetilde{\Gamma}_{33} & 0 & 0 & 0 & 0 \\
* & * & * & \widetilde{\Gamma}_{44} & 0 & 0 & 0 \\
* & * & * & * & -Q_{1} & 0 & C^{T} K^{T} \\
* & * & * & * & * & -Q_{2} & 0 \\
* & * & * & * & * & * & -2 I_{p}
\end{array}\right] \\
& \Phi_{12}=\left[\begin{array}{cccc}
A^{T} X & \widetilde{\Gamma}_{1,9} & 0 & \widetilde{\Gamma}_{1,11} \\
0 & \widetilde{A}^{T} Y & 0 & 0 \\
0 & \widetilde{\Gamma}_{3,9} & 0 & D^{T} U^{T} \\
0 & \widetilde{\Gamma}_{4,9} & 0 & U^{T} \\
0 & \widetilde{\Gamma}_{5,9} & 0 & \widetilde{\Gamma}_{5,11} \\
0 & 0 & 0 & 0 \\
0 & -\bar{\theta} U^{T} & 0 & -U^{T}
\end{array}\right], \\
& \Phi_{13}=\left[\begin{array}{ccccc}
0 & 0 & 0 & E^{T} X & E^{T} Y \\
0 & 0 & 0 & 0 & 0 \\
0 & 0 & 0 & G^{T} X & G^{T} Y \\
0 & 0 & 0 & 0 & 0 \\
0 & 0 & 0 & 0 & 0 \\
0 & 0 & 0 & 0 & 0 \\
0 & 0 & 0 & 0 & 0
\end{array}\right], \\
& \Phi_{22}=\operatorname{diag}\left\{-X,-Y,-\theta_{1}^{2} X,-\theta_{1}^{2} Y\right\} \text {, } \\
& \Phi_{23}=\left[\begin{array}{ccccc}
0 & 0 & 0 & 0 & 0 \\
\widetilde{\Gamma}_{9,12} & \widetilde{\Gamma}_{9,13} & \widetilde{\Gamma}_{9,14} & 0 & 0 \\
0 & 0 & 0 & 0 & 0 \\
U & U & U & 0 & 0
\end{array}\right] \text {, } \\
& \Phi_{33}=\operatorname{diag}\left\{-\varepsilon_{1} I_{p},-\varepsilon_{2} I_{p},-\varepsilon_{3} I_{p},-X,-Y\right\} \text {, } \\
& \widetilde{\Gamma}_{11}=-\bar{P}+\bar{L}^{T} \bar{L}+\varepsilon_{1} \bar{C}^{T} K_{1}^{T} \Delta^{T} \Delta K_{1} \bar{C}, \\
& \widetilde{\Gamma}_{1,9}=A^{T} Y-\widehat{A}^{T} Y+(\bar{\theta}-1) C^{T} K_{1}^{T} \widehat{B}^{T} Y \text {, } \\
& \widetilde{\Gamma}_{1,11}=A^{T} Y-W^{T}+(\bar{\theta}-1) C^{T} K_{1}^{T} U^{T}, \\
& \widetilde{\Gamma}_{22}=-Y+L^{T} L, \\
& \widetilde{\Gamma}_{33}=-\gamma^{2} I_{r}+\varepsilon_{2} D^{T} \Delta \Delta D, \\
& \widetilde{\Gamma}_{3,9}=(\bar{\theta}-1) D^{T} U^{T}, \\
& \widetilde{\Gamma}_{4,4}=-2 I_{p}+\varepsilon_{3} \Delta \Delta, \\
& \widetilde{\Gamma}_{4,9}=(\bar{\theta}-1) U^{T},
\end{aligned}
$$




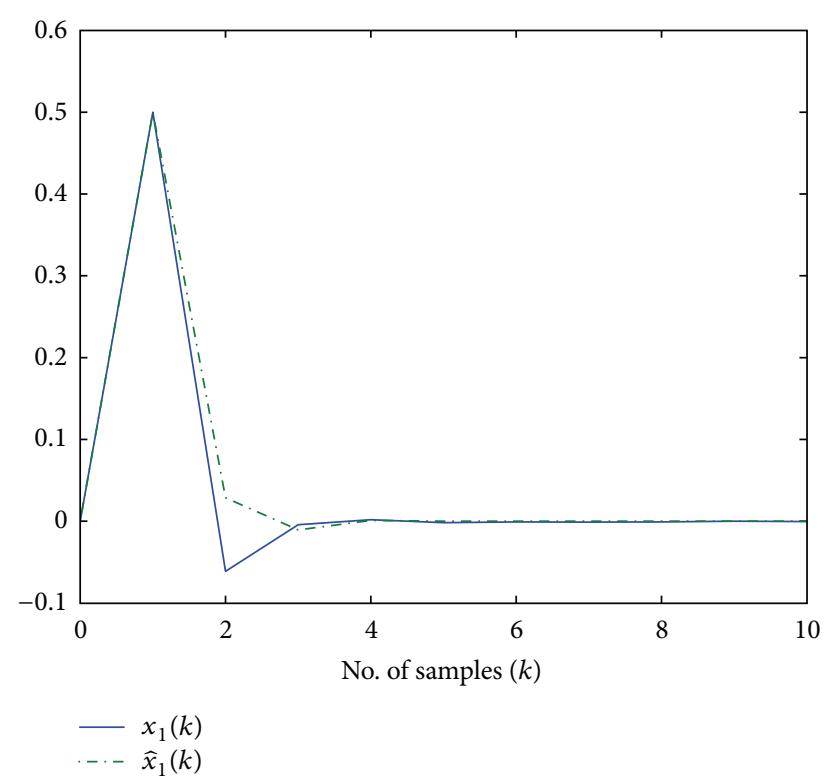

Figure 2: $x_{1}(k)$ and $\widehat{x}_{1}(k)$.

$$
\begin{gathered}
\widetilde{\Gamma}_{5,9}=-\bar{\theta} C^{T} K_{1}^{T} U^{T}, \\
\widetilde{\Gamma}_{5,11}=-C^{T} K_{1}^{T} Y, \\
\widetilde{\Gamma}_{9,12}=\widetilde{\Gamma}_{9,13}=\widetilde{\Gamma}_{9,14}=-\bar{\theta} U,
\end{gathered}
$$

then the $H_{\infty}$ filtering problem is solved by the filter (13). Furthermore, the filter gains are given by

$$
\widehat{A}=Y^{-1} W, \quad \widehat{B}=Y^{-1} U .
$$

Proof. The matrix condition (22) in Theorem 4 can be rewritten as

$$
\left[\begin{array}{cccccccc}
\Gamma_{11} & 0 & \bar{C}^{T} K^{T} & 0 & 0 & 0 & \bar{A}_{1}^{T} & \bar{A}_{2}^{T} \\
* & \Gamma_{22} & 0 & 0 & 0 & 0 & \bar{B}_{1}^{T} & 0 \\
* & * & -2 I_{p} & 0 & 0 & 0 & -\bar{H}_{1}^{T} & \bar{H}_{2}^{T} \\
* & * & * & -\bar{Q} & 0 & \bar{C}^{T} K^{T} & \bar{A}_{4}^{T} & A_{3}^{T} \\
* & * & * & * & -\gamma^{2} I_{m} & 0 & \bar{B}_{4}^{T} & \bar{B}_{3}^{T} \\
* & * & * & * & * & -2 I_{p} & \bar{H}_{4}^{T} & \bar{H}_{3}^{T} \\
* & * & * & * & * & * & -\bar{P}^{-1} & 0 \\
* & * & * & * & * & * & * & -\theta_{1}^{2} \bar{P}^{-1}
\end{array}\right]
$$

where

$$
\begin{gathered}
M_{1}=\left[0,0,0,0,0,0, \bar{H}_{1}^{T}, \bar{H}_{2}^{T}\right]^{T}, \\
N_{1}=\left[K_{1} \bar{C}, 0,0, K_{1} \bar{C}, 0,0,0,0\right], \\
N_{2}=[0, D, 0,0, D, 0,0,0]^{T}, \\
N_{3}=[0,0, I, 0,0, I, 0,0]^{T} .
\end{gathered}
$$

In the light of Lemma 5, it is shown that the condition (41) holds if the following condition holds:

$$
\left[\begin{array}{ccccccccccc}
\Gamma_{11} & 0 & \bar{C}^{T} K^{T} & 0 & 0 & 0 & \bar{A}_{1}^{T} & \bar{A}_{2}^{T} & 0 & 0 & 0 \\
* & \Gamma_{22} & 0 & 0 & 0 & 0 & \bar{B}_{1}^{T} & 0 & 0 & 0 & 0 \\
* & * & -2 I_{p} & 0 & 0 & 0 & -\bar{H}_{1}^{T} & \bar{H}_{2}^{T} & 0 & 0 & 0 \\
* & * & * & -Q & 0 & \bar{C}^{T} K^{T} & \bar{A}_{4}^{T} & \bar{A}_{3}^{T} & 0 & 0 & 0 \\
* & * & * & * & -\gamma^{2} I_{m} & 0 & \bar{B}_{4}^{T} & \bar{B}_{3}^{T} & 0 & 0 & 0 \\
* & * & * & * & * & -2 I_{p} & \bar{H}_{4}^{T} & \bar{H}_{3}^{T} & 0 & 0 & 0 \\
* & * & * & * & * & * & -\bar{P}^{-1} & 0 & (\bar{\theta}-1) \bar{H}_{2} & (\bar{\theta}-1) \bar{H}_{2} \\
* & * & * & * & * & * & * & -\theta_{1}^{2} \bar{P}^{-1} & \bar{H}_{2} & \bar{H}_{2} & \bar{H}_{2} \\
* & * & * & * & * & * & * & * & -\varepsilon_{1} I_{m} & 0 & 0 \\
* & * & * & * & * & * & * & * & * & -\varepsilon_{2} I_{m} & 0 \\
* & * & * & * & * & * & * & * & * & * & -\varepsilon_{3} I_{m}
\end{array}\right]<0 .
$$




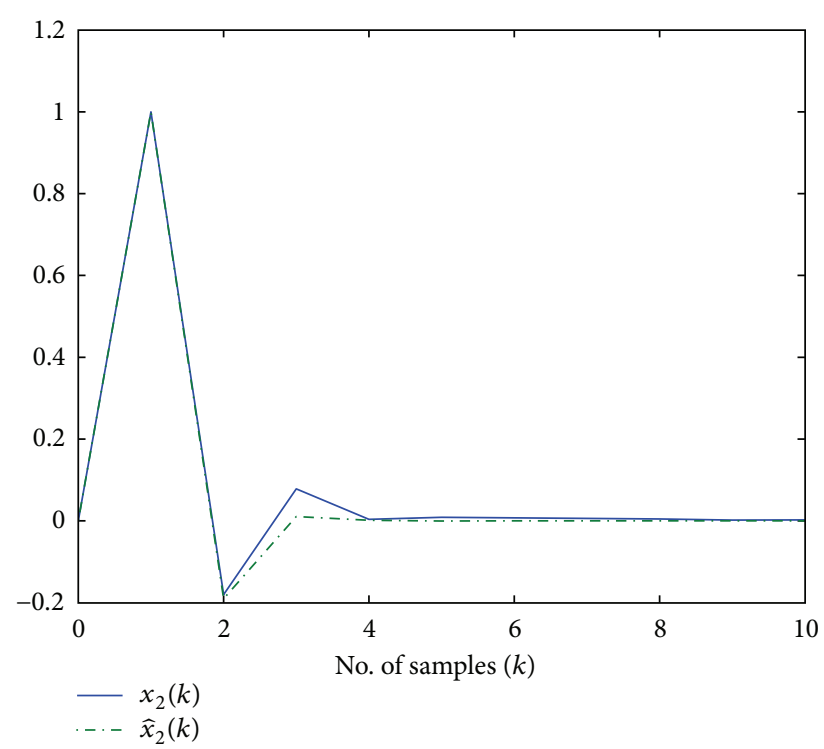

Figure 3: $x_{2}(k)$ and $\widehat{x}_{2}(k)$.

Multiply diag $\left\{I, I, I, I, I, I, P_{1}, P_{1}, I, I, I\right\}$ and its transpose on the left side and the right side of (43), respectively, and let $W=Y \widehat{A}, U=Y \widehat{B}$, one can obtain the condition (38). This means that if there exist scalars $\varepsilon_{1}>0, \varepsilon_{2}>0, \varepsilon_{3}>0$ such that the LMI condition (38) holds, then the error system (17) is stochastically stable, and the $H_{\infty}$ performance (32) is guaranteed. This completes the proof.

Remark 7. The LMI condition (38) of Theorem 6 is not conservative, since the system matrix $A$ has been supposed to be stable.

\section{Simulation}

We consider the system (1) with the following data: $n=3$, $m=2$, and $p=2, r=2$. For the logarithmic quantizer (7), the quantizer densities are chosen as $\rho_{1}=0.6667$ and $\rho_{2}=$ 0.7391 . The initial quantizer points are chosen as $\eta_{1}^{(0)}=40$ and $\eta_{2}^{(0)}=40$. It can be calculated that $\delta_{1}=0.2$ and $\delta_{2}=0.15$. The random communication delay parameters are selected as $\bar{\theta}=0.6$, and $\theta_{1}=\sqrt{\bar{\theta}(1-\bar{\theta})}=0.4899$. The saturation parameter matrices are selected as follows:

$$
K_{1}=\left[\begin{array}{cc}
0.6 & 0 \\
0 & 0.7
\end{array}\right], \quad K_{2}=\left[\begin{array}{cc}
0.8 & 0 \\
0 & 0.8
\end{array}\right]
$$

and $\phi\left(y_{k}\right)=\left(K_{1}+K_{2}\right) / 2 y_{k}+\left(K_{2}-K_{1}\right) / 2 \sin \left(y_{k}\right)$.
Besides, the model parameters are given as follows:

$$
\begin{gathered}
A=\left[\begin{array}{ccc}
-0.3 & 0 & 0.01 \\
-0.59 & -0.24 & 0.02 \\
0.1 & -0.06 & -0.68
\end{array}\right], \quad B=\left[\begin{array}{c}
-0.202 \\
0.383 \\
0.139
\end{array}\right], \\
C=\left[\begin{array}{ccc}
-0.2 & -0.1 & -0.2 \\
0.5 & 0.2 & 0.21
\end{array}\right], \quad D=\left[\begin{array}{c}
0.1 \\
0.47
\end{array}\right], \\
E=\left[\begin{array}{ccc}
-0.12 & -0.11 & 0.38 \\
0.11 & 0.64 & -0.18 \\
-0.31 & -0.63 & -0.6
\end{array}\right], \\
G=\left[\begin{array}{c}
-0.13 \\
0.11 \\
0.051
\end{array}\right], \quad L=\left[\begin{array}{ccc}
0.1 & 0.09 & 0.1 \\
0.05 & 0.05 & 0.05
\end{array}\right] .
\end{gathered}
$$

Without loss of generality, we assume that the noises $v(k)$ in system (1) have the following form:

$$
v(k)=\frac{1}{0.1+k^{2}},
$$

and it can be checked that $v(k)$ satisfies the constraint (2). Solving the LMI condition (38), one can obtain the following solutions:

$$
\begin{gathered}
X=\left[\begin{array}{ccc}
3.6195 & 3.3264 & 2.2784 \\
3.3264 & 10.8295 & 2.5230 \\
2.2784 & 2.5230 & 7.4548
\end{array}\right], \\
Y=\left[\begin{array}{lll}
4.3244 & 1.5752 & 1.6206 \\
1.5752 & 2.3707 & 0.2978 \\
1.6206 & 0.2978 & 2.3496
\end{array}\right], \\
W=\left[\begin{array}{lll}
-1.3882 & -0.4611 & -0.7446 \\
-1.1207 & -0.0697 & -0.4972 \\
-0.4623 & -0.1005 & -1.4395
\end{array}\right], \\
U=\left[\begin{array}{ll}
0.0118 & 0.0050 \\
0.0469 & 0.1449 \\
0.0390 & 0.0963
\end{array}\right] .
\end{gathered}
$$

The filter gain are then calculated as follows:

$$
\begin{gathered}
\widehat{A}=\left[\begin{array}{ccc}
-0.1855 & -0.1492 & 0.1803 \\
-0.3463 & 0.0632 & -0.2408 \\
-0.0249 & 0.0521 & -0.7065
\end{array}\right], \\
\widehat{B}=\left[\begin{array}{cc}
-0.0165 & -0.0580 \\
0.0277 & 0.0909 \\
0.0245 & 0.0695
\end{array}\right] .
\end{gathered}
$$

The initial condition is selected as $x(0)=\left[\begin{array}{lll}1.5 & 0 & -1\end{array}\right]^{T}$, $e(0)=\left[\begin{array}{lll}-0.5 & 1 & 1.5\end{array}\right]^{T}$, and the quantizer parameter $\eta_{0}$ is selected as $\eta_{0}=50$. The trajectories of plant states $x_{k}$ and its estimates are shown in Figures 2, 3, and 4; the comparisons of the unquantized saturated outputs $\phi\left(y_{k}\right)$ and quantized saturated outputs $q\left(\phi\left(y_{k}\right)\right)$ are shown in Figures 5 and 6; the trajectory of the error estimation signal $e_{z}(k)=z(k)-\widehat{z}(k)$ is illustrated in Figures 7 and 8 . It can be seen that the obtained state estimation is desirable. 


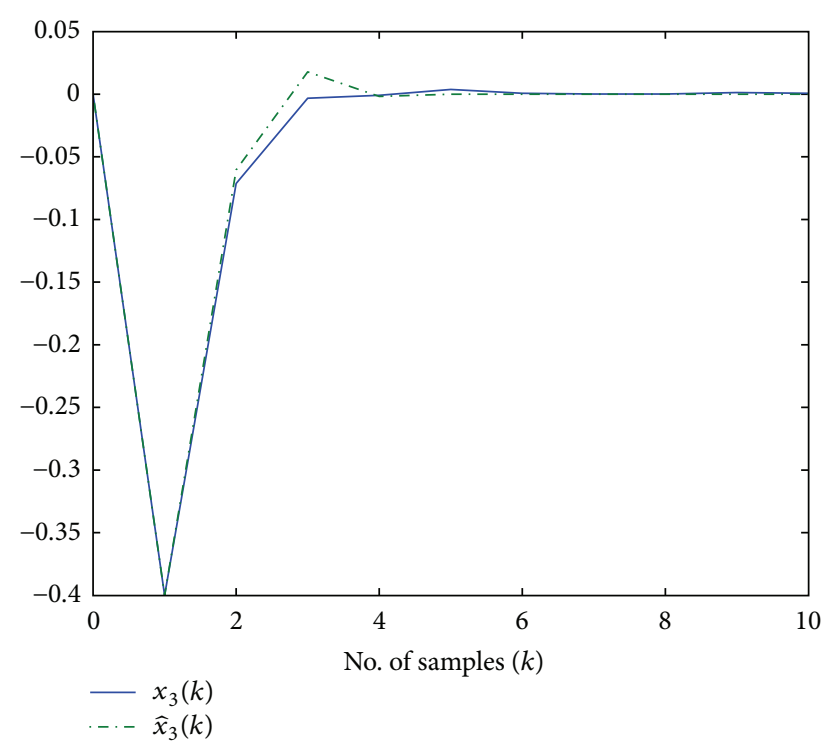

Figure 4: $x_{3}(k)$ and $\widehat{x}_{3}(k)$.

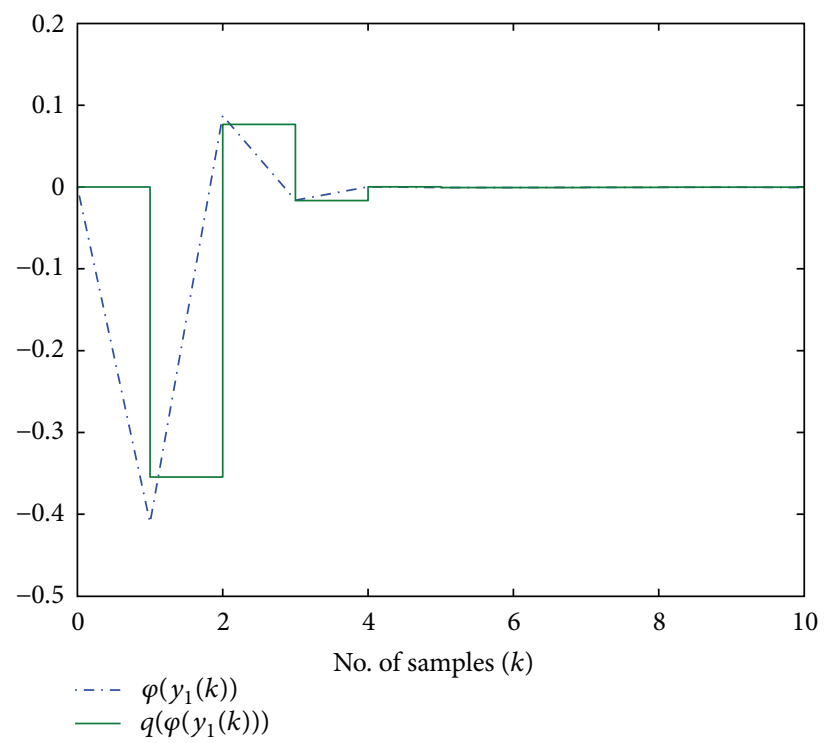

FIGURE 5: $\phi\left(y_{1}(k)\right)$ and quantized $\phi\left(y_{1}(k)\right)$.

\section{Conclusion}

In this paper, the $H_{\infty}$ filtering problem has been investigated for stochastic systems subject to sensor saturation over limited capacity channel. The plant under consideration is a class of stochastic systems with random noise depending on state and external disturbance. In this setting, the effects of sensor quantization, output logarithmic quantization, and networked-induced communication delay are taken into account simultaneously. The phenomenon of random communication delay is described by a Bernoulli type stochastic variable. Subsequently, the $H_{\infty}$ filter is designed for the

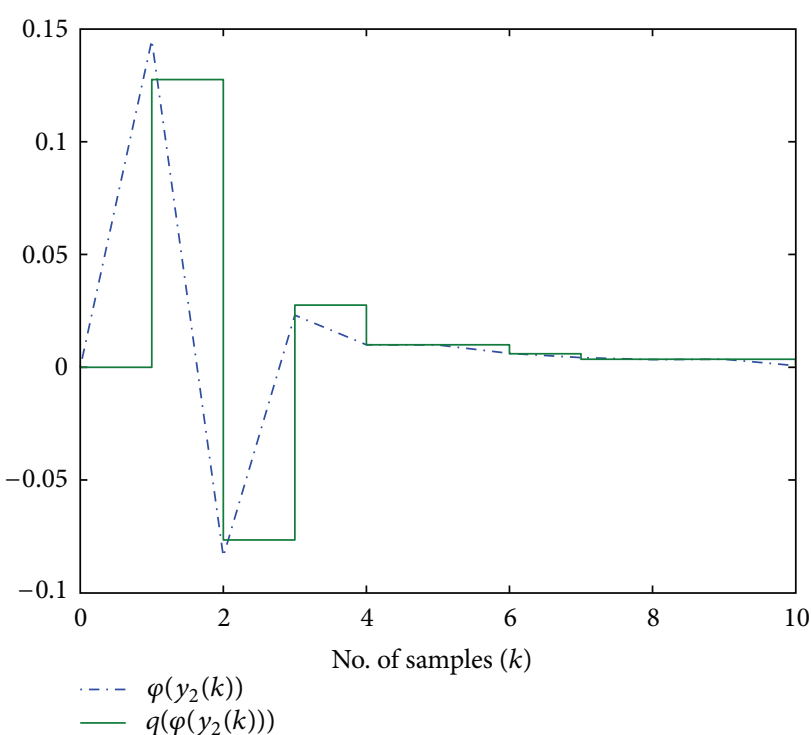

FIGURE 6: $\phi\left(y_{2}(k)\right)$ and quantized $\phi\left(y_{2}(k)\right)$.

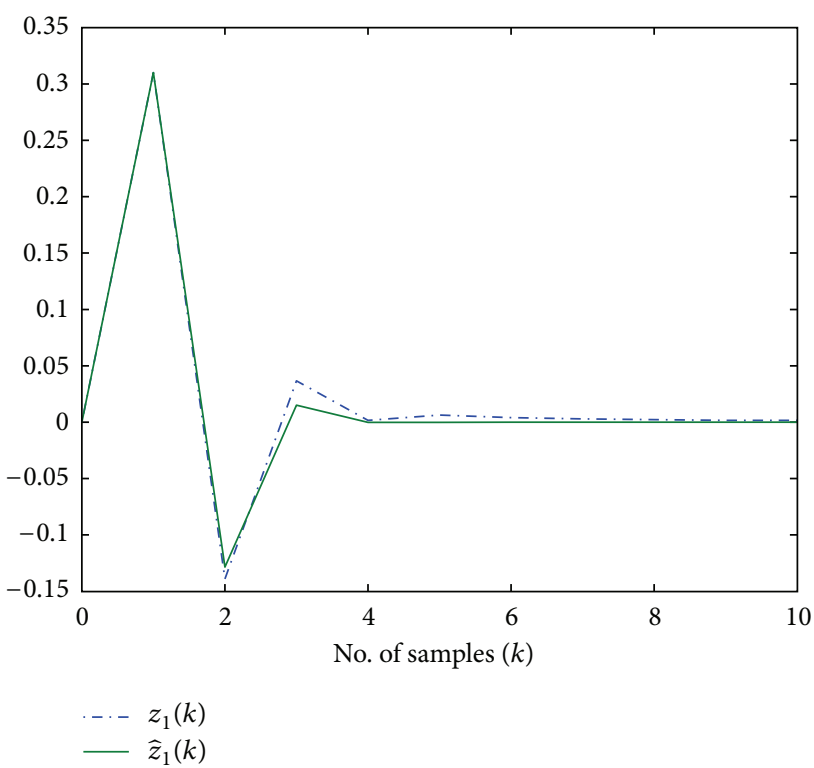

FIGURE 7: $z_{1}(k)$ and $\widehat{z}_{1}(k)$.

considered plant by employing the quantized output measurements, and sufficient conditions are established for the existence of the proposed filter.

\section{Acknowledgments}

This work is supported by the National High Technology Research and Development Program of China (no. 2012AA120601), National Natural Science Foundation of China (no. 11202044 and no. 11072044) and the Fundamental Research Funds for the Central Universities. 


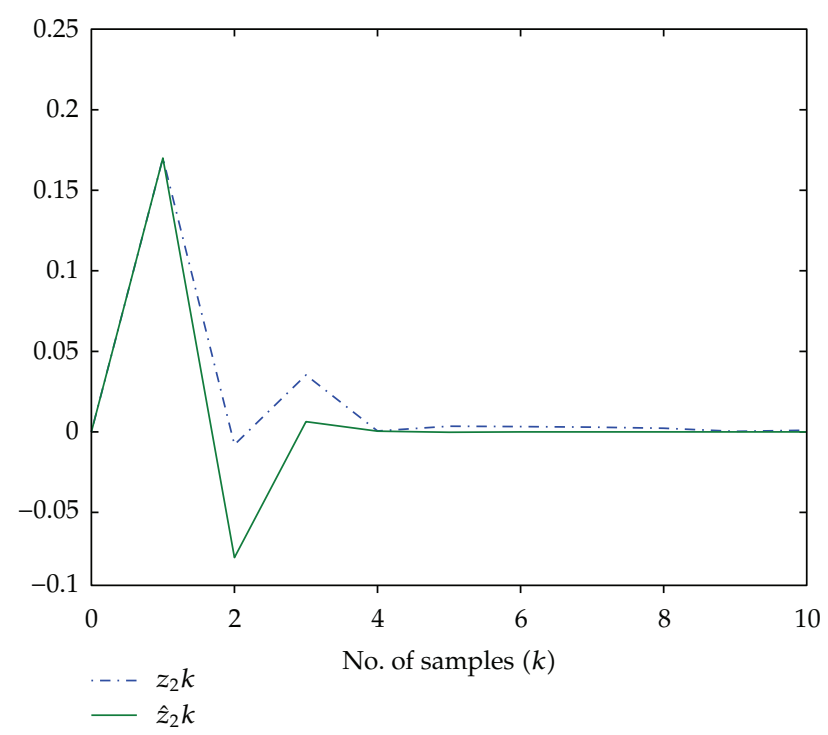

FIGURE 8: $z_{2}(k)$ and $\widehat{z}_{2}(k)$.

\section{References}

[1] M. Liu, D. W. C. Ho, and Y. Niu, "Stabilization of Markovian jump linear system over networks with random communication delay," Automatica, vol. 45, no. 2, pp. 416-421, 2009.

[2] M. Liu, D. W. C. Ho, and Y. Niu, "Robust filtering design for stochastic system with mode-dependent quantization output," IEEE Transactions on Signal Processing, vol. 58, no. 12, pp. 64106416, 2010.

[3] L. Wu, X. Su, P. Shi, and J. Qiu, "Model approximation for discrete-time state-delay systems in the T-S fuzzy framework," IEEE Transactions on Fuzzy Systems, vol. 19, no. 2, pp. 366-378, 2011.

[4] X. Su, P. Shi, L. Wu, and Y.-D. Song, "A novel approach to filter design for T-S fuzzy discrete-time systems with time-varying delay," IEEE Transactions on Fuzzy Systems, vol. 20, no. 6, Article ID 6189779, pp. 1114-1129, 2012.

[5] L. Wu and D. W. C. Ho, "Fuzzy filter design for nonlinear Itô stochastic systems with application to sensor fault detection," IEEE Transactions on Fuzzy System, vol. 17, no. 1, pp. 233-242, 2009.

[6] L. Wu and D. W. C. Ho, "Sliding mode control of singular stochastic hybrid systems," Automatica, vol. 46, no. 2, pp. 779783, 2010.

[7] L. Wu, P. Shi, H. Gao, and C. Wang, " $H_{\infty}$ filtering for $2 \mathrm{D}$ Markovian jump systems," Automatica, vol. 44, no. 7, pp. 1849$1858,2008$.

[8] L. Wu and W. X. Zheng, " $L_{2}-L_{\infty}$ control of nonlinear fuzzy itô stochastic delay systems via dynamic output feedback," IEEE Transactions on Systems, Man, and Cybernetics B, vol. 39, no. 5, pp. 1308-1315, 2009.

[9] L. Wu, D. W. C. Ho, and C. W. Li, "Sliding mode control of switched hybrid systems with stochastic perturbation," Systems \& Control Letters, vol. 60, no. 8, pp. 531-539, 2011.

[10] G. Wu and J. Zhang, "Reliable passivity and passification for singular Markovian systems," IMA Journal of Mathematical Control and Information, 2012.

[11] M. Liu, Q. Wang, and H. Li, "State estimation and stabilization for nonlinear networked control systems with limited capacity channel," Journal of the Franklin Institute, vol. 348, no. 8, pp. 1869-1885, 2011.

[12] S. Xu and T. Chen, " $H_{\infty}$ output feedback control for uncertain stochastic systems with time-varying delays," Automatica, vol. 40, no. 12, pp. 2091-2098, 2004.

[13] S. Endowed, "Recovery of signals distorted by sensor nonlinearity," Journal of Dynamic Systems, Measurement and Control, vol. 126, no. 4, pp. 848-859, 2004.

[14] R. Pearson, "Gray-box modeling of nonideal sensors," in Proceedings of the American Control Conference, vol. 6, pp. 44044409, Arlington, Va, USA, 2001.

[15] G. Kreisselmeier, "Stabilization of linear systems in the presence of output measurement saturation," Systems \& Control Letters, vol. 29, no. 1, pp. 27-30, 1996.

[16] E. Fridman and M. Dambrine, "Control under quantization, saturation and delay: an LMI approach," Automatica, vol. 45, no. 10, pp. 2258-2264, 2009.

[17] S. Xu, J. Lam, and T. Chen, "Robust $H_{\infty}$ control for uncertain discrete stochastic time-delay systems," Systems \& Control Letters, vol. 51, no. 3-4, pp. 203-215, 2004.

[18] Y. Niu, J. Lam, X. Wang, and D. W. C. Ho, "Observer-based sliding mode control for nonlinear state-delayed systems," International Journal of Systems Science, vol. 35, no. 2, pp. 139150, 2004.

[19] V. Kolmanovskii and A. Myshkis, Applied Theory of Functional Differential Equations, Kluwer Academic, Dordrecht, The Netherlands, 1992. 


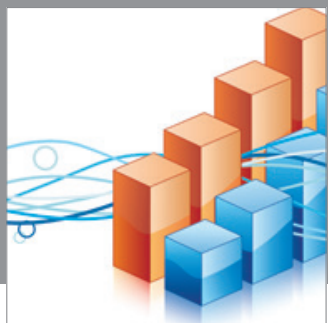

Advances in

Operations Research

mansans

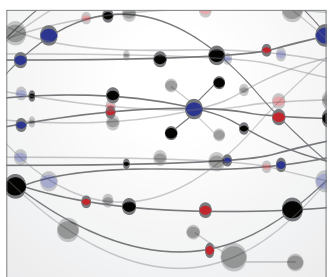

The Scientific World Journal
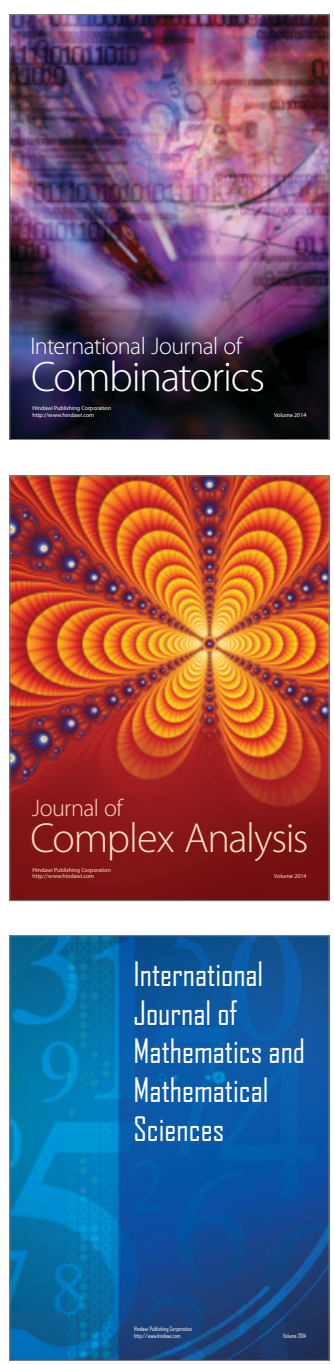
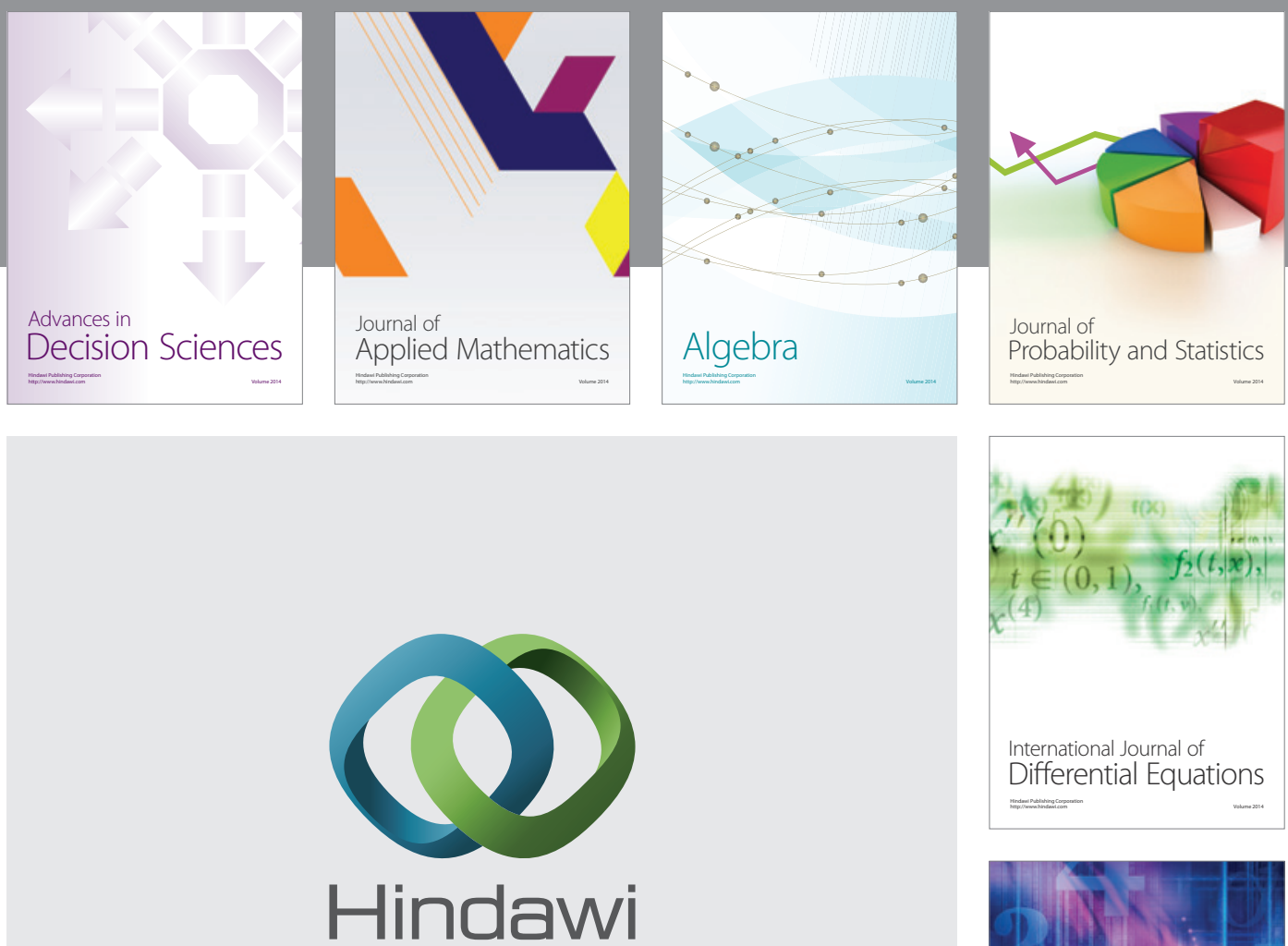

Submit your manuscripts at http://www.hindawi.com
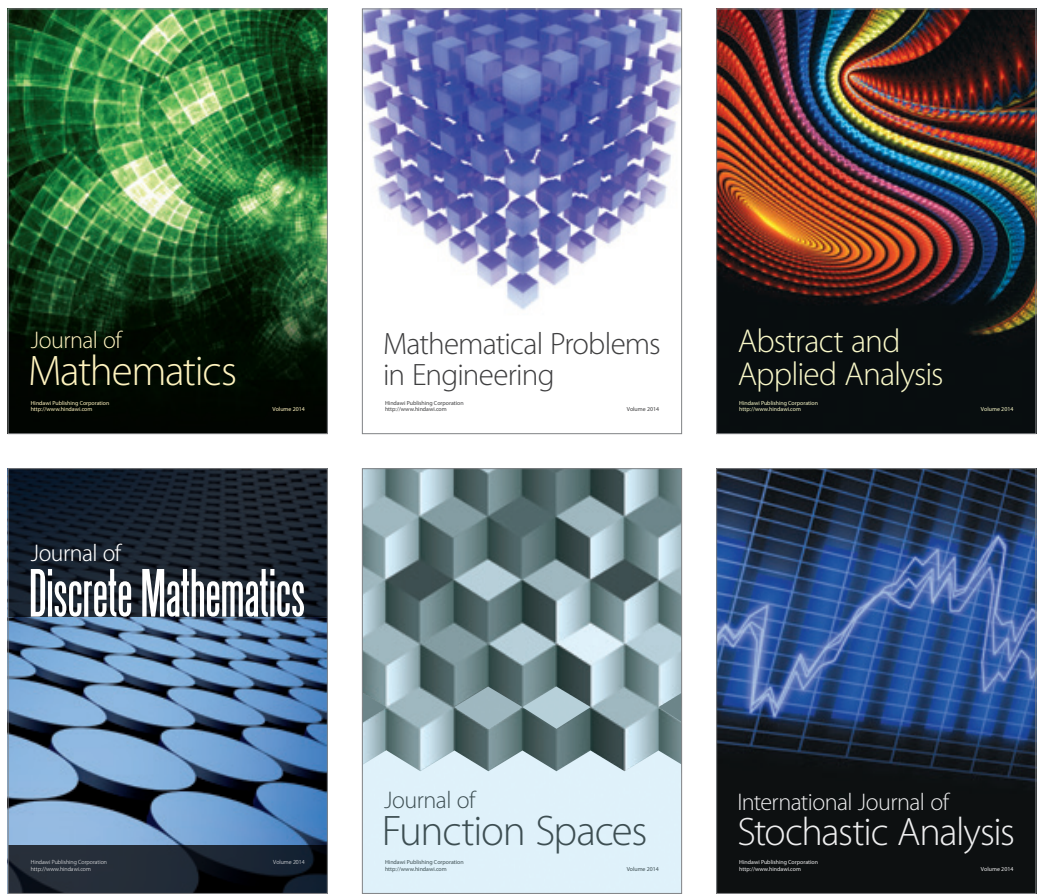

Journal of

Function Spaces

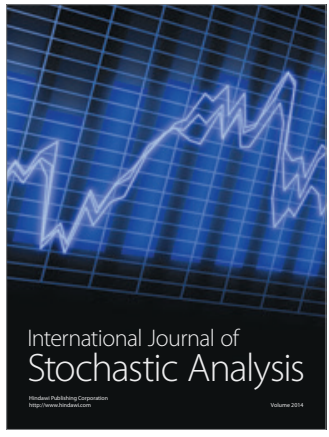

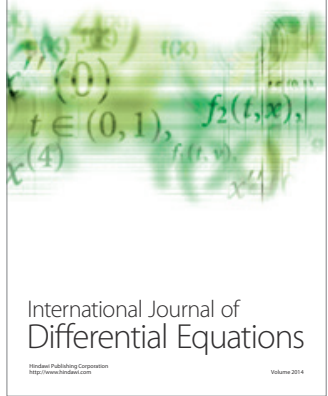
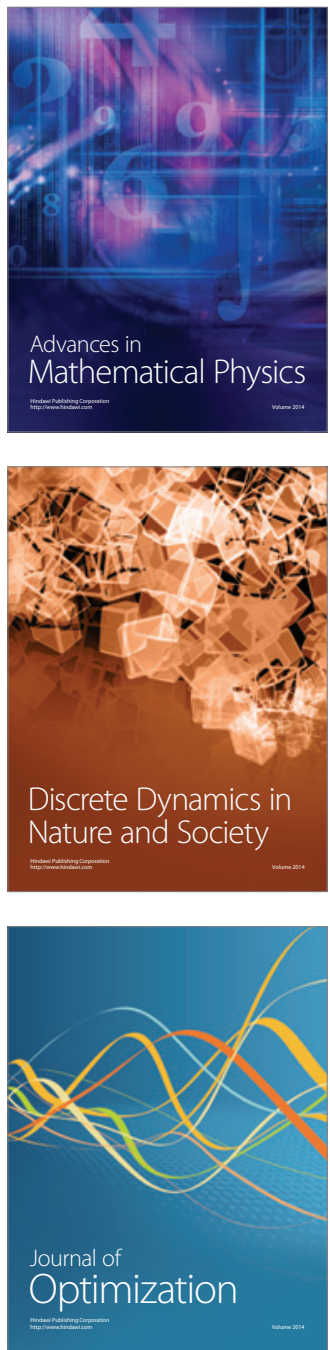